\title{
Comparison of Principal Component Analysis and Multivariate Curve Resolution-Alternating Least Squares Analysis of XPS Spectral Maps
}

\author{
F. B. Lavoie ${ }^{1}$, N. Braidy ${ }^{1}$, S. Biais ${ }^{2}$, R. Gosselin ${ }^{1}$ \\ ${ }^{1}$ Department of Chemical and Biotechnological Engineering, Université de Sherbrooke. Sherbrooke, \\ (QC) J1K 2R1 Canada \\ ${ }^{2}$ Centre de caractérisation des matériaux. Université de Sherbrooke. Sherbrooke, ( QC) J 1K 2R1 \\ Canada
}

Recent instrument developments of X-ray Photoelectron Spectroscopy (XPS) have made it possible to acquire multispectral images with a spatial resolution of $\sim 3 \mu \mathrm{m}$ without sacrificing the exceptional energy resolution of the technique. The simplest method to produce composite maps from a data cube is to assign to a channel the background-subtracted intensities of characteristic edges to a particular color channel. However, if overlapping peaks are present in the spectrum, such a strategy will fail to map the independent components. In addition, despite the recent instrument improvements, the signal remains weak, especially when approaching the lateral resolution limit. To compensate, lengthy (and expensive) acquisition times (several hours) are required to collect a statistically significant dataset, which can lead to beam-induced damage and spatial drift.

Principal Component Analysis (PCA) has emerged as an efficient and widespread method to process multispectral materials XPS images [1]. Unlike peak integration, it allows components having overlapping features on the energy scale to contribute separately to the phase map. Although PCA allows the efficient visualization of uncorrelated variables by removing the ones associated to noise, the principal components do not correspond to phases. Multivariate Curve Resolution Alternating Least Squares (MCR) is another method for multivariate data processing [2]. MCR is an iterative algorithm that generates the components by minimizing an objective function with a set of relevant constraints. The aim of this work is to compare the performance of PCA and MCR to identify and map the phases of an XPS map.

A test specimen made of $\mathrm{TiO}_{2} / \mathrm{Ti}_{2} \mathrm{O}_{3}$ circular ridges on $\mathrm{Ti}$ was produced using microfabrication techniques. Here, we deliberately chose a system for which the XPS peak of a metal and its oxide are known to significantly overlap in order to fully explore the sensitivity of the proposed methods. The spectrum image was collected with a dwell time of $300 \mathrm{~s} /$ frame using a XPS (Kratos Axis Ultra ${ }^{\text {DLD }}$ ) operating with a monochromatic $\mathrm{Al} \mathrm{K} \alpha$ anode and equipped with a delay-line detector. 181 frames of 256 pix $\times 256$ pix $(400 \mu \mathrm{m} \times 400 \mu \mathrm{m})$ were collected using a pass energy of $40 \mathrm{eV}$ which translates to an energy window of $\sim 3.6 \mathrm{eV}$, between $447 \mathrm{eV}$ and $465 \mathrm{eV}$ across the Ti $2 \mathrm{p}$ region. All the dataset were processed with MATLab®, using the PLS toolbox [3].

The maps of the first and second components of the PCA correlate spatially to Ti (Fig. 1a) and a Tioxide (Fig. 1b), respectively. As expected, the spectral signature of the components cannot be directly related to the reference spectra of $\mathrm{Ti}$ and Ti-oxide (Figs. 2c and 2d). The dataset was also processed using MCR using constraints of non-negativity of the intensity and closure (for a given pixel, the sum of all the components must be unity). The MCR-processed maps of Ti (Fig. 2a) and Ti-oxide (Fig. 2b) are also coherent with the model but are significantly sharper than the PCA-processed maps. In addition, the corresponding spectra of the MCR components are much closer to the references and can 
be readily interpreted. PCA and MCR were compared using 3 figures of merit (Table 1): i) fraction of variance captured ii) signal-to-noise ratio and iii) type I/II errors (false positive/false negative) of the assignation of the pixel to a given phase. On all counts, the MCR methods scores significantly better than the PCA algorithm.

Using a standard test sample, we demonstrated several advantages in terms of signal to noise ratio and accuracy of the MCR compared to PCA for the processing of XPS multispectral images. For a given accuracy and precision, a better post-processing tool for XPS enables one to shorten the acquisition time and avoid unnecessary beam damage. Further work will concentrate on comparing MCR to various clustering and denoising algorithm.

\section{References:}

[1] Artyushkova K. and Fulghum J.E. (2002) Surface and Interface Analysis, 33(3), 185-195.

[2] Piqueras S. et al. (2011) Analytica Chimica Acta, 705(1-2), 182-192

[3] http://www.eigenvector.com/software/pls_toolbox.htm_accessed February 13th, 2014

[4] Acknowledgments: We are grateful to GREEN-TPV and Université de Sherbrooke for funding this research.
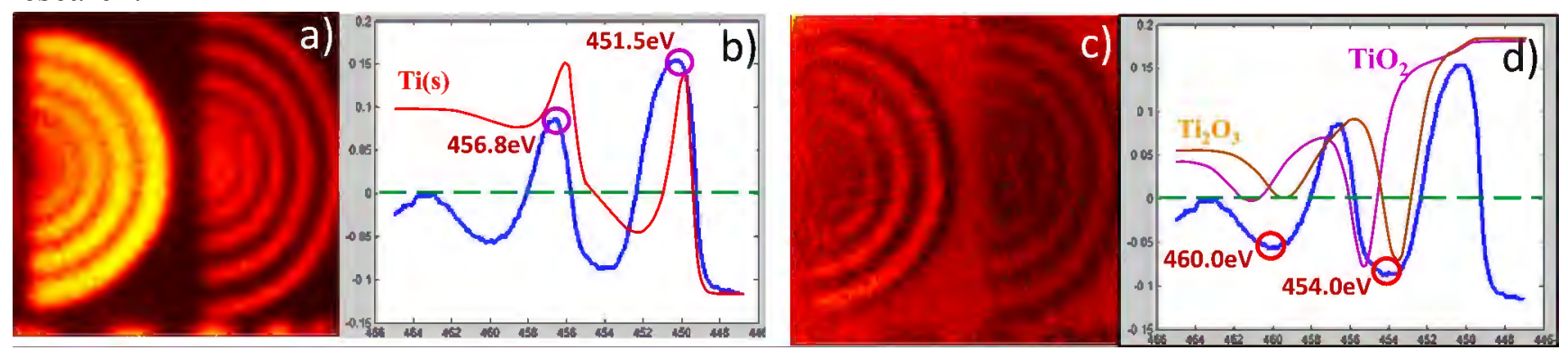

Fig. 1. Map (a) of component 1 computed using PCA and (b) corresponding spectra (blue solid curve) compared to the $\mathrm{Ti} 2 \mathrm{p}$ reference spectra (red curve). Component 2 shown (c) with corresponding spectrum (d), (blue line) with $\mathrm{TiO}_{2}$ and $\mathrm{Ti}_{2} \mathrm{O}_{3}$ reference spectra (magenta and orange lines). Image width: $400 \mu \mathrm{m}$
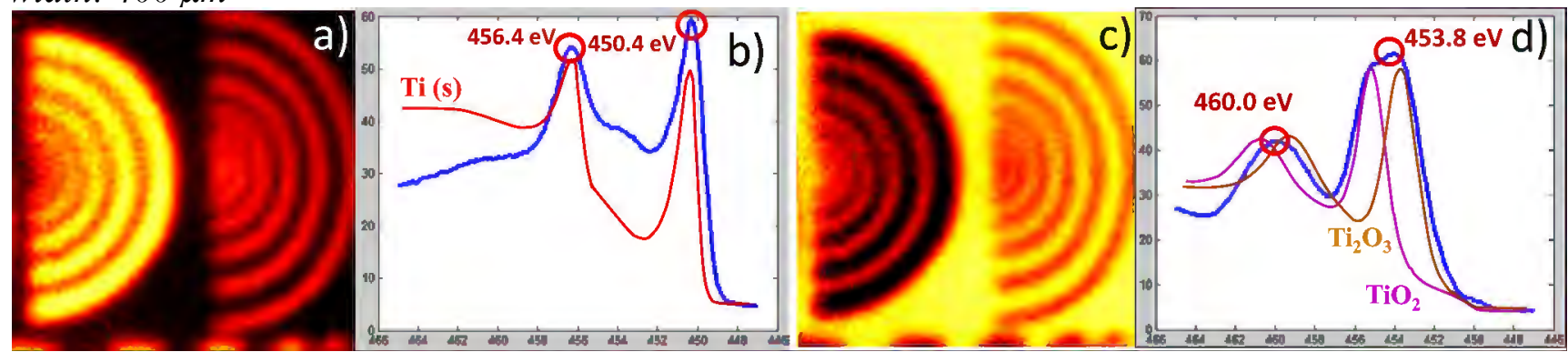

Fig. 2. Map (a) of component 1 computed using MCR and (b) corresponding spectra (blue solid curve) compared to the Ti $2 p$ reference spectra (red curve). Component 2 shown (c) with corresponding spectrum (d), (blue line) with $\mathrm{TiO}_{2}$ and $\mathrm{Ti}_{2} \mathrm{O}_{3}$ reference spectra (magenta and orange lines). Image width: $400 \mu \mathrm{m}$

Table 1. Figures of merit of PCA and MCR components1

\begin{tabular}{|ccccc|}
\hline & $\mathbf{P C A}_{1}$ & $\mathbf{P C A}_{2}$ & $\mathbf{M C R}_{1}$ & $\mathbf{M C R}_{2}$ \\
\hline Variance captured (\%) & 17.5 & 2.0 & 30.3 & 60.7 \\
\hline Signal to noise ratio (\%) & 15.0 & 2.3 & 24.0 & 23.5 \\
\hline Error types I \& II (\%) & 4.9 & 17.1 & 4.9 & 4.9 \\
\hline
\end{tabular}

